\title{
Effect of CD44 on differentiation of human amniotic mesenchymal stem cells into chondrocytes via Smad and ERK signaling pathways
}

\author{
YAN XU $^{1}$, YI-QING WANG ${ }^{1}$, AI-TONG WANG ${ }^{1}$, CHANG-YIN YU $^{2}$, YI LUO $^{1,2}$, \\ RU-MING LIU ${ }^{1,2}$, YU-JIE ZHAO ${ }^{3}$ and JIAN-HUI XIAO ${ }^{1,2}$ \\ ${ }^{1}$ Zunyi Municipal Key Laboratory of Medicinal Biotechnology, Institute of Medicinal Biotechnology, \\ ${ }^{2}$ Center for Translational Medicine and ${ }^{3}$ Department of Laboratory Medicine, Affiliated Hospital of \\ Zunyi Medical University, Zunyi, Guizhou 563003, P.R. China
}

Received July 12, 2019; Accepted February 28, 2020

DOI: $10.3892 / \mathrm{mmr} .2020 .11044$

\begin{abstract}
CD44 antigen (CD44) is a transmembrane protein found in cell adhesion molecules and is involved in the regulation of various physiological processes in cells. It was hypothesized that CD44 directly affected the chondrogenic differentiation of human amniotic mesenchymal stem cells (hAMSCs). In the present study, the expression of chondrocyte-associated factors was detected in the absence and presence of the antibody blocker anti-CD44 antibody during the chondrogenic differentiation of hAMSCs. Following inhibition of CD44 expression, the transcriptional levels of chondrocyte-associated genes SRY-box transcription factor 9, aggrecan and collagen type II $\alpha 1$ chain, as well as the production of chondrocyte markers type II collagen and aggrecan were significantly decreased in hAMSCs. Further investigation indicated that there was no significant change in total ERK1/2 expression following inhibition of CD44 expression; however, phosphorylated (p)-ERK1/2 expression was decreased. The expression of $\mathrm{p}-\mathrm{Smad} 2 / 3$ was also upregulated following CD44 inhibition. These data indicated that CD44 may affect the differentiation of hAMSCs into chondrocytes by regulating the Smad2/3 and ERK1/2 signaling pathway.
\end{abstract}

Correspondence to: Professor Jian-Hui Xiao, Zunyi Municipal Key Laboratory of Medicinal Biotechnology, Institute of Medicinal Biotechnology, Affiliated Hospital of Zunyi Medical University, 201 Dalian Road, Huichuan, Zunyi, Guizhou 563003, P.R. China

E-mail: jhxiao@zmu.edu.cn

Key words: CD44 antigen, human amniotic mesenchymal stem cells, chondrogenic differentiation, Smad signaling pathway, ERK signaling pathway

\section{Introduction}

Osteoarthritis is a very common degenerative joint disease involving highly diseased connective tissue and is a leading cause of disability. Furthermore, the damaged articular cartilage has limited self-repairing ability, making it difficult to cure $(1,2)$. With the development of cell therapy, attempts have been made to expand chondrocytes in vitro to repair cartilage defects; however, the expansion of chondrocytes in vitro causes decreased proliferative ability and dedifferentiation (3). Mesenchymal stem cells (MSCs) exhibit the potential for self-renewal and multi-directional differentiation and serve an important role in tissue regeneration $(4,5)$. Therefore, inducing the differentiation of MSCs into chondrocytes has become an important approach to repair damaged cartilage $(6,7)$. An inflammatory milieu may induce chondrocyte apoptosis, resulting in cartilage destruction in patients with cartilage degenerative diseases, such as rheumatoid arthritis or osteoarthritis (8). Notably, MSCs can differentiate into chondrocytes under inflammatory conditions to repair damaged tissue (9). This indicates the potential of MSCs in the treatment of cartilage diseases. Human amniotic mesenchymal stem cells (hAMSCs) exhibit the characteristics of MSCs and differentiate into adipocyte-, osteoblast- and chondrocyte-like cells, exhibiting low immunogenicity and immunoregulatory function. Therefore, they are an ideal cell resource for stem cell therapy and tissue engineering $(10,11)$. Furthermore, transplantation of hAMSCs may enhance bone strength and decrease the incidence of fractures in mice (12). They exhibited stronger cartilage repair capability compared with human bone marrow mesenchymal stem cells and chondrocytes (13). However, the regulatory mechanism underlying the differentiation of hAMSCs into chondrocytes remains to be elucidated.

CD44 antigen (CD44) is cell surface protein that can bind to various ligands, including extracellular matrix components, growth factors and cytokines, and regulates cell signaling (14). CD44 is essential for maintaining cartilage homeostasis (15). A previous study demonstrated that hyaluronic acid promotes the differentiation of adipose-derived mesenchymal stem cells into chondrocytes by promoting CD44 clustering (16). 
In addition, chondrocytes with high expression of CD44 exhibited stronger chondrogenic capacity (17). However, the specific role of CD44 in the differentiation of MSCs, including hAMSCs, into chondrocytes remains to be elucidated.

The mitogen-activated protein kinase (MAPK) signaling pathway serves an important role in cell proliferation, differentiation, migration, senescence and apoptosis (18). A previous study reported that the MAPK signaling pathway, including ERK1/2, JNK and p38 MAPK molecules, is activated in chondrocytes (19). Activation of ERK signaling pathway can enhance the ability of MSCs to differentiate into osteoblasts and cardiomyocytes $(20,21)$. In addition, the ERK1/2 signaling pathway regulates chondrocyte differentiation and cartilage formation in in vitro (22). However, the inhibition of ERK signaling activation delays the maturation of hypertrophic chondrocytes and leads to a slower rate of fracture healing $(23,24)$.

In the present study, it was hypothesized that hAMSCs would differentiate into chondrocytes by enhancing CD44 expression. Therefore, the effect of CD44 on chondrogenic differentiation of hAMSCs was evaluated. In addition, ERK phosphorylation and Sox-9 levels in hAMSCs chondrogenesis were examined.

\section{Materials and methods}

Cell culture. The study and use of the human amniotic membrane were approved by the Ethics Committee of Affiliated Hospital of Zunyi Medical University. A total of 20 pregnant women were recruited for the study and these healthy pregnant women met the following eligibility criteria: Between 18 and 35 years of age; and without hepatitis B or C, or HIV infection. The sample was collected after obtaining written informed consent from the women and the samples were collected between May 2018 and March 2019. The primary hAMSCs were isolated from term placental amnion of healthy pregnant women according to the method previously described by Zhang et al (25) and hAMSCs were cultured at $37^{\circ} \mathrm{C}$ in low glucose-Dulbecco's modified Eagle's medium (LG-DMEM; Gibco; Thermo Fisher Scientific, Inc.) supplemented with $10 \%$ fetal bovine serum (FBS; Gibco; Thermo Fisher Scientific, Inc.), $1 \%$ non-essential amino acids (NEAA; Gibco; Thermo Fisher Scientific, Inc.) and $10 \mathrm{ng} / \mathrm{ml}$ basic fibroblast growth factor (PeproTech, Inc.), and 1\% L-GlutaMAX. Culture medium was replaced by fresh medium after every 3 days. When the cells reached $80 \%$ confluency, the harvested cells were then passaged. Cells at passage 2 (P2) were used for the present study.

In vitro chondrocyte differentiation of hAMSCs. The P2 hAMSCs at the logarithmic growth phase were inoculated into 6-well plates at a dose of $2 \times 10^{5}$ cells/well. The cells were divided into 2 experimental groups; the negative control group (NC) and the positive drug group (PG). The NC was cultured in complete high-glucose (HG)-DMEM (Gibco; Thermo Fisher Scientific, Inc.) supplemented with 10\% FBS, $1 \%$ GlutaMAX, $1 \%$ NEAA and $55 \mu \mathrm{mol} / 1 \beta$-mercaptoethanol. The PG was cultured in chondrogenic induction medium, which consisted of complete HG-DMEM, $10 \mathrm{ng} / 1$ transforming growth factor (TGF) $\beta$-3 (PeproTech, Inc.), $1 \times 10^{-7} \mathrm{~mol} / 1$ dexamethasone
(Sigma-Aldrich; Merck KGaA) and $50 \mathrm{mg} / \mathrm{l}$ vitamin C (Beijing Solarbio Science \& Technology Co., Ltd.). The medium was changed after every 3 days and chondrocyte-associated markers, including type II collagen and aggrecan, were detected on day 7 following induction.

Immunocytochemistry staining. Immunocytochemistry staining was used to detect the expression of vimentin (MSCs surface marker protein) and type II collagen (chondrocytes marker) in hAMSCs. When the P2 hAMSCs reached $80 \%$ confluency, the cells were digested with trypsin and washed 3 times with Dulbecco's PBS (D-PBS) for 5 min each time and then treated with $0.3 \%$ Triton X-100 for 15 min at room temperature to increase cell membrane permeability, and then washed 3 times with D-PBS. The harvested cells were incubated with 5\% BSA (Beijing Solarbio Science \& Technology Co.,Ltd.) at room temperature to block non-specific antigen interaction. Following blocking for $30 \mathrm{~min}$, primary antibodies, including anti-vimentin (cat. no. GM072504; Gene Tech Co., Ltd.), anti-cytokeratin 19 (cat. no. GM088804; Gene Tech Co., Ltd.) and anti-collagen type II (cat. no. ab34712; Abcam) were added to the cells. Then, the cells were incubated at $4^{\circ} \mathrm{C}$ overnight and washed 3 times with D-PBS for 5 min each time. The negative control was treated with the same amount of D-PBS but no primary antibody. Finally, the labeled cells were treated with the secondary antibody (1:500; cat. no. SA00001-1; ProteinTech Group, Inc.) and incubated at $37^{\circ} \mathrm{C}$ for $30 \mathrm{~min}$. Following washing 3 times with D-PBS, the color reaction was developed using diaminobenzidine (at room temperature for $30 \mathrm{sec}$ ) under a light microscope (magnification, $\mathrm{x} 100)$ and the reaction was stopped using distilled water. Nuclei were counterstained with hematoxylin for $1 \mathrm{~min}$ at room temperature.

Flow cytometry analysis. For the phenotypic characterization of hAMSCs, the third passage hAMSCs at the logarithmic growth phase were harvested and labeled with different antibodies for hMSC-specific markers [5'-nucleotidase (CD73), thy-1 membrane glycoprotein (CD90) and endoglin (CD105)] using BD stemflow Human MSC analysis kit (cat. no. 562245; BD Biosciences) for flow cytometry analysis (26). In brief, the 3rd-passage hAMSCs were collected following trypsin digestion, washed twice with D-PBS containing 0.1\% BSA, resuspended in D-PBS containing $0.1 \%$ BSA, adjusted to a density of $1 \times 10^{6}$ cells $/ \mathrm{ml}$ and then incubated with the corresponding antibody for $1 \mathrm{~h}$ in the dark. Following washing again with D-PBS containing 0.1\% BSA, the cell suspension was centrifuged at $100 \mathrm{x} \mathrm{g}$ at room temperature for $5 \mathrm{~min}$ and the supernatant was discarded. Finally, the labeled cells were analyzed by flow cytometry using Cell Quest software version 5.1 after fixation at $4^{\circ} \mathrm{C}$ overnight with $1 \%$ paraformaldehyde (PFA).

Toluidine blue staining. Toluidine blue staining was used to analyze the secretion of aggrecan during the chondrogenic differentiation of hAMSCs. Briefly, the cells were washed 3 times with D-PBS for 5 min each time on day 7 after chondrogenic induction and then were fixed with 4\% PFA for $20 \mathrm{~min}$ at room temperature. After washing 3 times for $5 \mathrm{~min}$ each time with D-PBS, $0.1 \%$ toluidine blue dye solution (Beijing Solarbio 
Science \& Technology Co., Ltd.) was added to the cells. The cells were stained at room temperature for $30 \mathrm{~min}$, washed with D-PBS again and observed under an inverted microscope (magnification, x100).

Inhibition of CD44. The P2 hAMSCs in the logarithmic growth phase were inoculated into 6-well plates at a dose of $2 \times 10^{5}$ cells/well. The cells were divided into 4 experimental groups: The NC group; the positive drug group (PG); anti-CD44 antibody (cat. no. A3D8; GeneTex, Inc.) inhibition group $\mathrm{NC}+\mathrm{A} 3 \mathrm{D} 8$; and $\mathrm{PG}+\mathrm{A} 3 \mathrm{D} 8$. After $24 \mathrm{~h}$ of inoculation, the anti-CD44 antibody $(2 \mu \mathrm{g} / \mathrm{ml})$ was used to inhibit the expression of CD44 molecules in hAMSCs in the incubator at $37^{\circ} \mathrm{C}$ for 3 or 7 days. The medium was replaced with fresh medium after every 3 days. The expression of CD44 and chondrocyte-associated markers was detected on day 7 .

Reverse transcription-quantitative polymerase chain reaction (RT-qPCR). The P2 hAMSCs at the logarithmic growth phase were seeded into 6 -well plates at $2 \times 10^{5}$ cells/well. On day 7 of chondrogenic differentiation of hAMSCs, hAMSCs were collected from the 6-well plate in each experimental group. RNAiso Plus (Takara Biotechnology Co., Ltd.) was used to extract total RNA from the cells, following the manufacturer's instructions. Total RNA ( $1 \mu \mathrm{g}$ per $20 \mu \mathrm{l}$ reaction volume) was reverse transcribed into cDNA using PrimeScript RT reagent kit (Takara Biotechnology Co., Ltd.), according to the manufacturer's protocols. qPCR was performed and monitored using SYBR Premix Ex Taq II (Takara Biotechnology Co., Ltd.) and a quantitative real-time PCR system (Bio-Rad Laboratories Inc.). The cDNA samples (3 $\mu \mathrm{l}$ samples in a total volume of $15 \mu \mathrm{l}$ per reaction) were analyzed for the genes of interest, including $C D 44$, collagen type II $\alpha 1$ chain (Col2al), SRY-box transcription factor 9 (Sox9), aggrecan (Acan), ERK1, ERK2 and $\beta$-actin. The relative mRNA transcriptional level of each target gene was calculated from the threshold cycle $(\mathrm{Ct})$ value of each PCR product and normalized to the expression of $\beta$-actin using the comparative $2^{-\Delta \Delta \mathrm{Cq}}$ method (27). Thermocycling conditions were as follows: i) Initial denaturation at $95^{\circ} \mathrm{C}$ for $30 \mathrm{sec}$; ii) Denaturation at $95^{\circ} \mathrm{C}$ for $30 \mathrm{sec}$; iii) Annealing at $60^{\circ} \mathrm{C}$ for $30 \mathrm{sec}$; iv) Elongation at $60^{\circ} \mathrm{C}$ for $30 \mathrm{sec}$; v) Final extension for 39 cycles. Each experiment was repeated at least 3 times. The primer sequences used are listed in Table I.

Western blot analysis. On day 7 of chondrogenic differentiation of hAMSCs, total proteins were extracted from the cells using RIPA lysis buffer (cat. no. R0020; Beijing Solarbio Science $\&$ Technology Co., Ltd.) and protein concentration was determined by the BCA method. Then, total proteins (10 $\mu \mathrm{l} / \mathrm{lane})$ were separated by SDS-PAGE gel (10\%) and transferred to the PVDF membranes, which were blocked with 5\% BSA for $1 \mathrm{~h}$ at room temperature. Following this, PVDF membranes were incubated with overnight at $4^{\circ} \mathrm{C}$ with primary antibodies, including ERK1+ERK2 antibody (1:1,000; cat. no. ab17942; Abcam), phosphorylated (p)-ERK1/2 antibody $(1: 1,000$; cat. no. 9101S; Cell Signaling Technology, Inc.), Smad2/3 antibody (1:1,000; cat. no. 3102S; Cell Signaling Technology, Inc.) and p-Smad2/3 antibody (1:1,000; cat. no. 8828S; Cell Signaling Technology, Inc.). Then, membranes were incubated
Table I. Primer sequences for reverse transcription-quantitative PCR.

\begin{tabular}{|c|c|c|}
\hline Gene & Primer sequence $\left(5^{\prime} \rightarrow 3^{\prime}\right)$ & $\begin{array}{c}\text { Length of } \\
\text { product (bp) }\end{array}$ \\
\hline \multirow[t]{2}{*}{ Col2al } & F: CAACACTGCCAACGTCCAGAT & 121 \\
\hline & R: TCTTGCAGTGGGCTGCCTTAT & \\
\hline \multirow[t]{2}{*}{ Sox9 } & F: GCGGAGGAAGTCCGTGAAGA & 82 \\
\hline & R: GAAGATGGCGTTGGGGGAGA & \\
\hline \multirow[t]{2}{*}{ Acan } & F: GTGCCTATCAGGACAAGGTCT & 167 \\
\hline & R: GATGCCTTTCACGACGACTTC & \\
\hline \multirow[t]{2}{*}{$C D 44$} & F: CTGCCGCTTTGCAGGTGTA & 109 \\
\hline & R: CATTGTGGGCAAGGTGCTATT & \\
\hline \multirow[t]{2}{*}{ ERK1 } & F: CTACACGCAGTTGCAGTACAT & 157 \\
\hline & R: CAGCAGGATCTGGATCTCCC & \\
\hline \multirow[t]{2}{*}{$E R K 2$} & F: TCTGGAGCAGTATTACGACCC & 134 \\
\hline & R: CTGGCTGGAATCTAGCAGTCT & \\
\hline
\end{tabular}

F, forward; R, reverse; Col2a1, collagen type II $\alpha 1$ chain; Sox9, SRY-box transcription factor 9; Acan, aggrecan.

with horseradish peroxidase conjugated-secondary antibody (1:5,000; cat. no. SA00001-2; ProteinTech Group, Inc.) for $2 \mathrm{~h}$ at room temperature. Absin ECL hypersensitive luminescent solution (cat. no. abs920; Absin) was used for exposure. The gray value of the strips was analyzed by ImageJ version 1.8.0 (National Institutes of Health) and the relative expression of the proteins was calculated. Protein phosphorylation was calculated as the ratio of phosphorylated to total protein expression. These data was normalized to a basal value of 1.0.

Statistical analysis. The data were expressed as the means \pm standard errors of the mean. Statistical significance was evaluated by unpaired t-tests in GraphPad Prism 8.0 (GraphPad Software, Inc.). $\mathrm{P}<0.05$ was considered to indicate a statistically significant difference.

\section{Results}

Morphological characteristics and phenotypic identification of hAMSCs. The primary culture of hAMSCs isolated was observed after $24 \mathrm{~h}$ of incubation, revealing that hAMSCs were adherent cells with irregular cell morphology, as shown in Fig. 1A. Following 3 subcultures, the third generation of hAMSCs was uniform in morphology with fibrous, spindle-shaped cells with spiral-like growth (Fig. 1A). Immunocytochemistry results indicated that hAMSCs highly expressed the MSC marker vimentin, but not the epithelial cell marker keratin, type 1 cytoskeletal 19 (CK19; Fig. 1B). To further verify the biological characteristics of hAMSCs, the surface molecular markers of MSCs were examined by flow cytometry. The results indicated that hAMSCs highly expressed surface molecular markers of MSCs, including CD90 (98.14\%), CD73 (99.95\%), CD105 (86.23\%), CD44 (99.89\%) and integrin $\beta-1$ (99.96\%); however, they did not express cell surface molecules of hematopoietic stem cells, such 
A
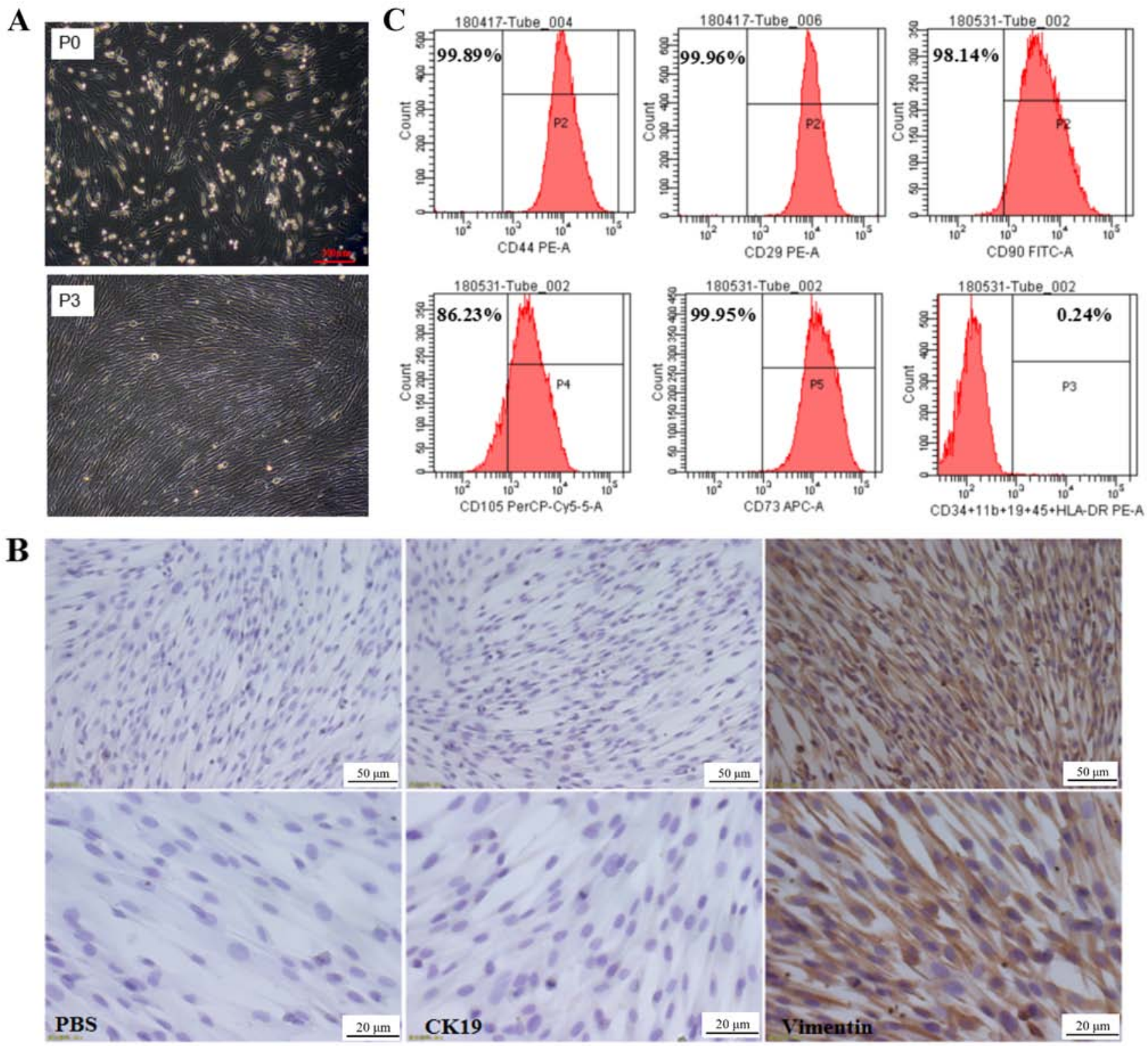

Figure 1. Morphological characteristics and phenotypic identification of hAMSCs. (A) Morphological characteristics of the primary hAMSCs (P0) and the third passage hAMSCs (P3). Scale bar, $200 \mu \mathrm{m}$. (B) Expression of CK19 and vimentin was assessed in hAMSCs. Brown staining denoted vimentin and CK19. The nuclei were counterstained with hematoxylin (blue). Upper panel, scale bar=50 $\mu \mathrm{m}$. Scale bar, $20 \mu \mathrm{m}$. (C) Flow cytometric analysis of hAMSCs. hAMSCs, human amniotic mesenchymal stem cells; PE, phycoerythrin; FITC, fluorescein isothiocyanate; CK19, keratin, type 1 cytoskeletal 19.

as hematopoietic progenitor cell antigen CD34, receptor-type tyrosine-protein phosphatase $\mathrm{C}$, integrin $\alpha$-M, B-lymphocyte antigen CD19 and HLA class II histocompatibility antigen $\gamma$ chain (Fig. 1C). This was consistent with the MSC accreditation standards recommended by the International Society for Cellular Therapy (28) and thus the isolated hAMSCs exhibited typical characteristics of MSCs.

Suppression of CD44 inhibits differentiation of hAMSCs into chondrocytes. To validate the role of CD44 in the differentiation of hAMSCs into chondrocytes, the extracellular matrices of chondrocyte, including type II collagen and aggrecan, were examined by immunocytochemistry staining in the presence or absence of A3D8, a CD44 inhibitor. On day 7 of chondrogenic differentiation of hAMSCs, type II collagen was observed in the PG; however, following inhibition with A3D8, no such differentiation was observed in the
PG (Fig. 2A). This indicated that the inhibition of CD44 could inhibit the formation of type II collagen. Concomitantly, the production of aggrecan showed similar results. The inhibition of CD44 significantly decreased the production of aggrecan in the PG (Fig. 2B). These results indicated that suppression of CD44 could inhibit the differentiation of hAMSCs into chondrocytes.

Inhibition of CD44 decreases the expression of chondrocyte-associated genes. To further verify the effect of CD44 on chondrocyte differentiation of hAMSCs, the transcriptional levels of chondrocyte-associated genes, $\operatorname{Sox} 9, \operatorname{Col} 2 \alpha 1$ and Acan, were detected by RT-qPCR following suppression of CD44. As shown in Fig. 3A, $2 \mu \mathrm{g} / \mathrm{ml}$ anti-CD44 antibody, A3D8, could effectively decrease the transcriptional level of CD44 in hAMSCs. In addition, the transcriptional levels of Sox9, Col2 $\alpha 1$ and Acan were significantly decreased in 
A

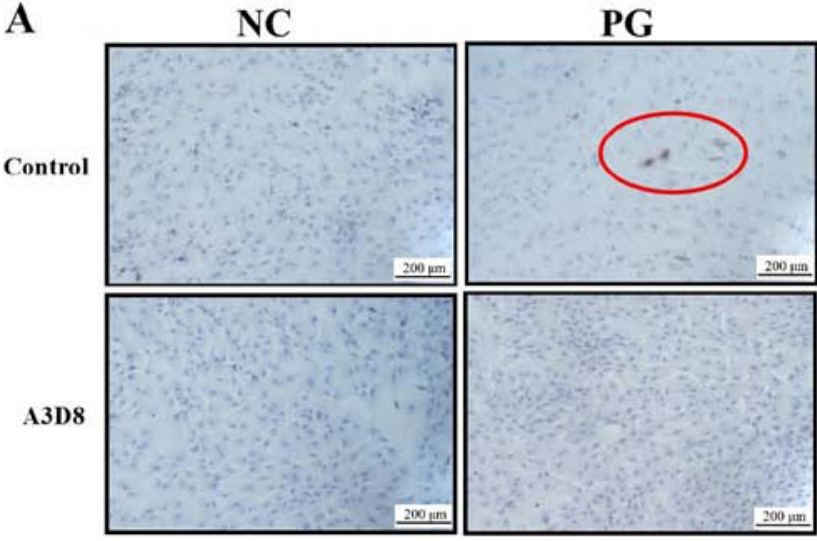

B

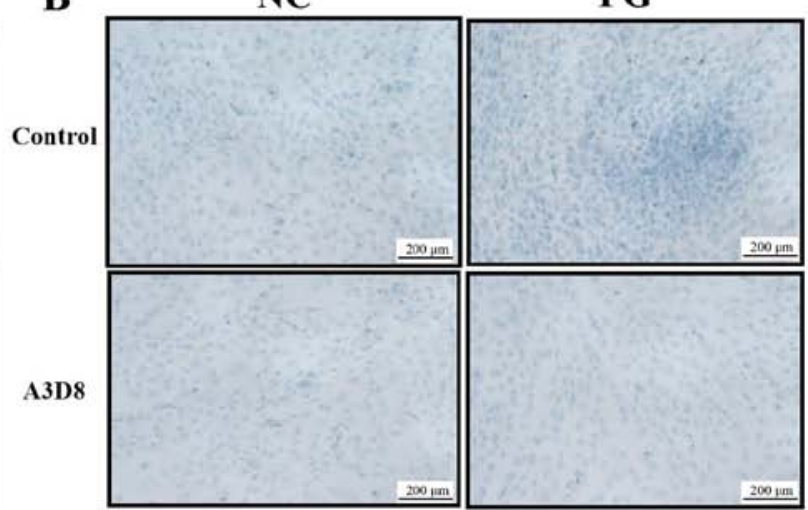

Figure 2. Suppression of CD44 may decrease the formation of type II collagen and aggrecan. (A) Expression of type II collagen in NC and PG groups in P3 hAMSCs on day 7 of treatment with $2 \mu \mathrm{g} / \mathrm{ml}$ A3D 8 by immunocytochemistry staining. The red circle indicates type II collagen; the nuclei were counterstained with hematoxylin (blue). (B) Toluidine blue staining for formation of aggrecan in NC and PG groups on day 7 of treatment with $2 \mu \mathrm{g} / \mathrm{ml}$ A3D8. Blue staining denotes aggrecan. Scale bar, $200 \mu \mathrm{m}$. NC, negative control group; PG, positive drug group; hAMSCs, human amniotic mesenchymal stem cells.

A
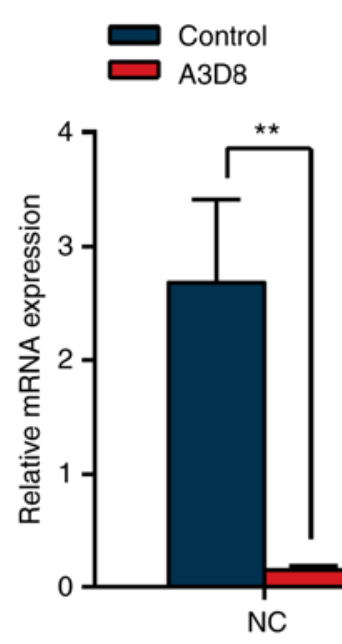

C

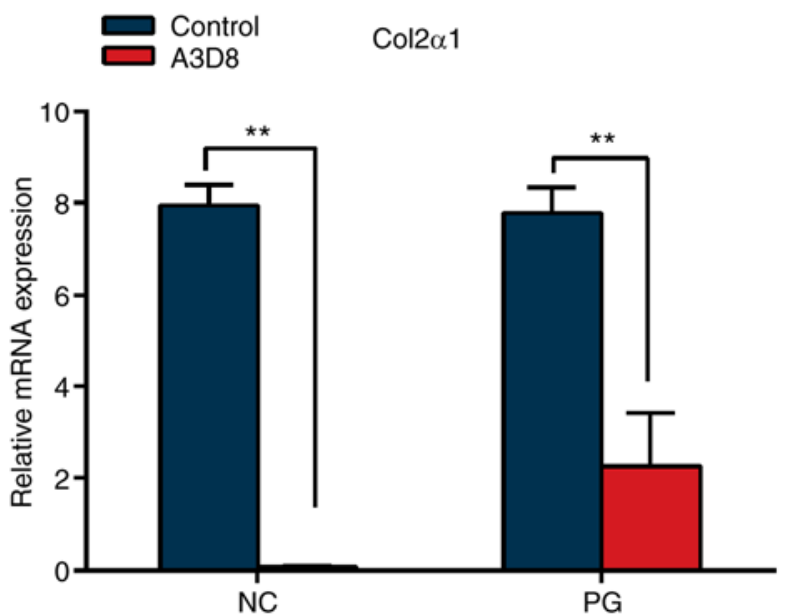

B

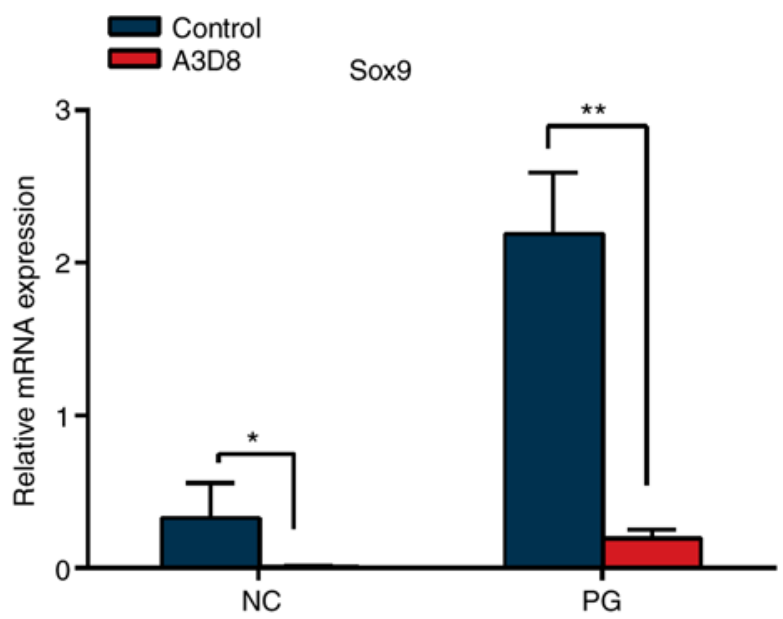

D

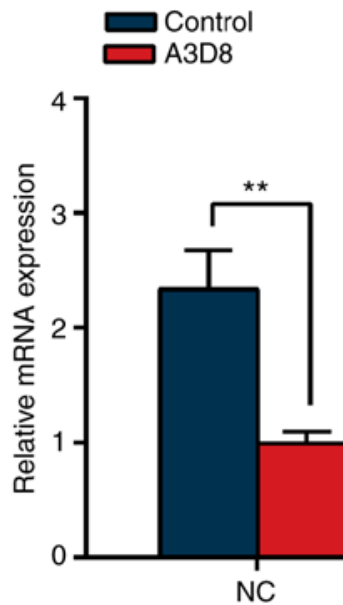

Acan

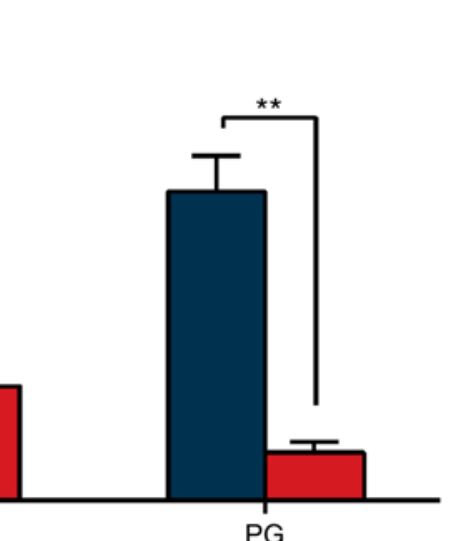

Figure 3. Suppression of CD44 decreases the expression of chondrocyte-associated genes. Relative expression levels of (A) CD44, (B) Sox9, (C) Co2 al and (D) Acan in P3 hAMSCs were detected by reverse transcription-quantitative polymerase chain reaction on day 7 after treatment with A3D8. Data were presented as mean \pm standard errors of the mean $(n=3)$. ${ }^{*} \mathrm{P}<0.05$ and ${ }^{* *} \mathrm{P}<0.01$. CD, cluster of differentiation; hAMSCs, human amniotic mesenchymal stem cells; Sox9, SRY-box transcription factor 9; Col2a1, collagen type II $\alpha 1$ chain; Acan, aggrecan; NC, negative control group; PG, positive drug group.

the presence of A3D8 (Fig. 3B-D). These data indicated that suppression of CD44 significantly attenuated the chondrocyte differentiation potential of hAMSCs.
Suppression of CD44 inhibits ERK1/2 pathway. To investigate whether CD44 could regulate chondrocyte differentiation potential of hAMSCs by ERK signaling pathway, the relative 


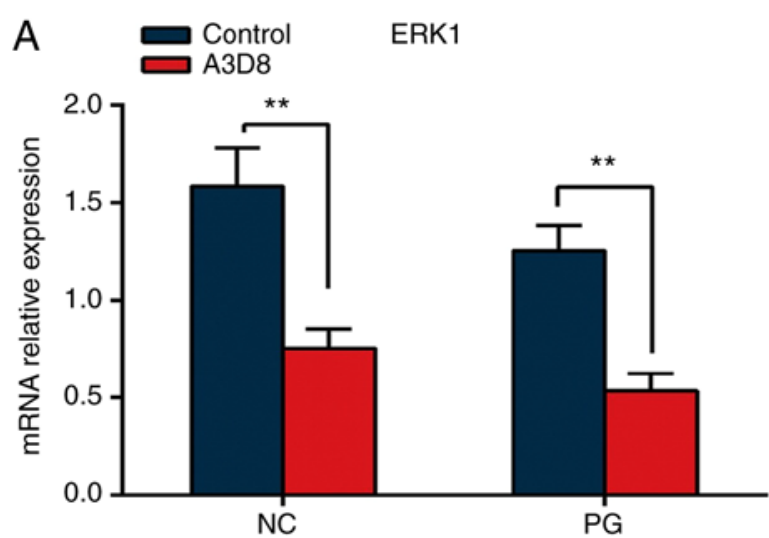

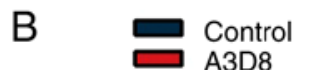

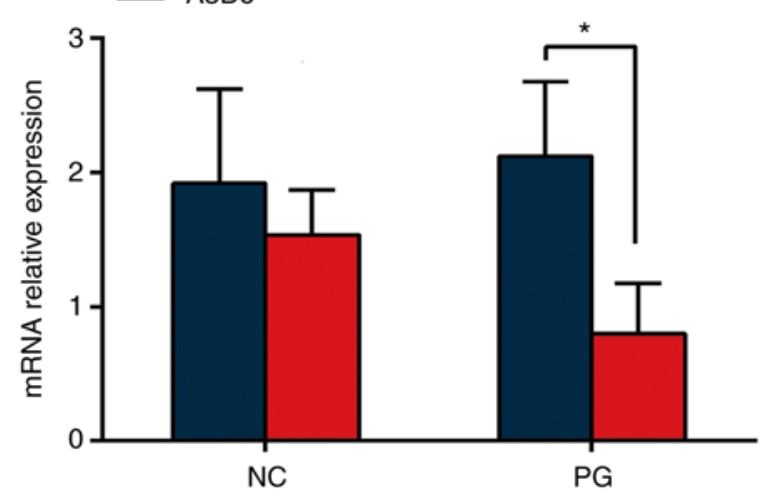

D ${ }_{\text {A3D8 }}^{\text {Control }} \quad$ p-ERK $1 / 2$

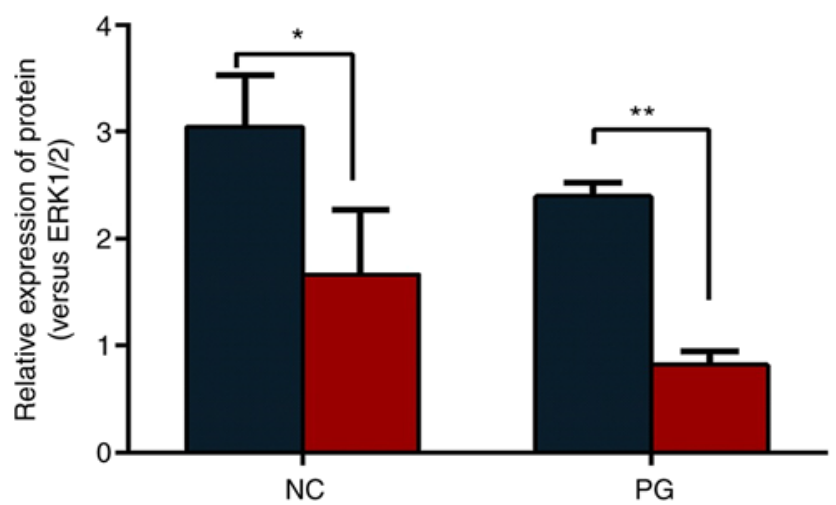

Figure 4. Suppression of CD44 inhibits ERK1/2 signaling during the chondrogenic differentiation of hAMSCs. Change in transcriptional levels of (A) ERK1 and (B) ERK2 in P3 hAMSCs after 7 days of inhibition of CD44 with $2 \mu \mathrm{g} / \mathrm{ml}$ A3D8. (C) Expression of p-ERK1/2 and ERK1/2 on day 7 after treatment with A3D8. (D) Ratio of p-ERK1/2 to ERK1/2 protein on day 7 after treatment with A3D8. Data were presented as mean \pm standard errors of the mean (n=3). ${ }^{*} \mathrm{P}<0.05$ and ${ }^{* *} \mathrm{P}<0.01$. CD, cluster of differentiation; hAMSCs, human amniotic mesenchymal stem cells; $\mathrm{p}-$, phosphorylated; t-, total; NC, negative control group; PG, positive drug group.

expression levels of ERK1 and ERK2 were examined in the presence or absence of A3D8 during the differentiation of hAMSCs into chondrocytes. Following the addition of A3D8, the transcriptional levels of ERK1 and ERK2 were significantly decreased (Fig. 4A and B). To further verify that CD44 regulated the chondrogenic differentiation of hAMSCs by the ERK1/2 signaling pathway, the expression of p-ERK1/2 protein following treatment with A3D8 was examined. As shown in Fig. 4C, no significant change in the expression of total ERK1/2 protein compared with the normal group was observed. However, the ratio of p-ERK1/2 to total ERK1/2 protein was significantly decreased following inhibition of CD44 (Fig. 4C and D). These results indicated CD44 may regulate the chondrocyte differentiation potential of hAMSCs via the ERK1/2 signaling pathway.

Suppression of CD44-induced Smad2/3 signaling activation. The TGF- $\beta /$ Smad signaling pathway serves an important role in chondrogenesis and there was cross-talk between Smad2/3 and ERK signaling in the regulation of MSC chondrogenesis (29). Therefore, the expression levels of Smad2/3 and p-Smad2/3 proteins were detected in the presence or absence of A3D8 by western blot analysis. As shown in Fig. 5, p-Smad2/3 expression was increased on day 1 and 3 when CD44 expression was inhibited by A3D8 (Fig. 5A and D) and the ratio of p-Smad2/3 to total Smad2/3 was also significantly upregulated following CD44 inhibition (Fig. 5C and F), particularly on day 3. However, the expression of $\operatorname{Smad} 2 / 3$ was decreased on day 3 following CD44 blocking (Fig. 5E). This indicated that CD44 regulated the phosphorylation of Smad2/3 directly, not via regulation of $\mathrm{Smad} 2 / 3$ expression. In addition, the expression of $\mathrm{p}-\mathrm{Smad} 2 / 3$ on day 3 was significantly decreased compared with on day 1 in the PG group (Fig. 5A and D). Therefore, the effect of CD44 on the differentiation of hAMSCs into chondrocytes not only regulated the ERK1/2 signal but also the Smad2/3 signal.

\section{Discussion}

CD44 is a principal receptor for hyaluronic acid, a major extracellular matrix component, and hyaluronic acid exhibits great potential for regulating the proliferation and differentiation of stem cells (30). Of note, articular chondrocytes also express the receptor CD44 and disruption of CD44 has profound effect on cartilage metabolism (31). Although CD44 serves a critical role in maintaining cartilage homeostasis, it remains unclear whether CD44 can regulate the chondrogenic differentiation of stem cells. The present study first demonstrated 
A
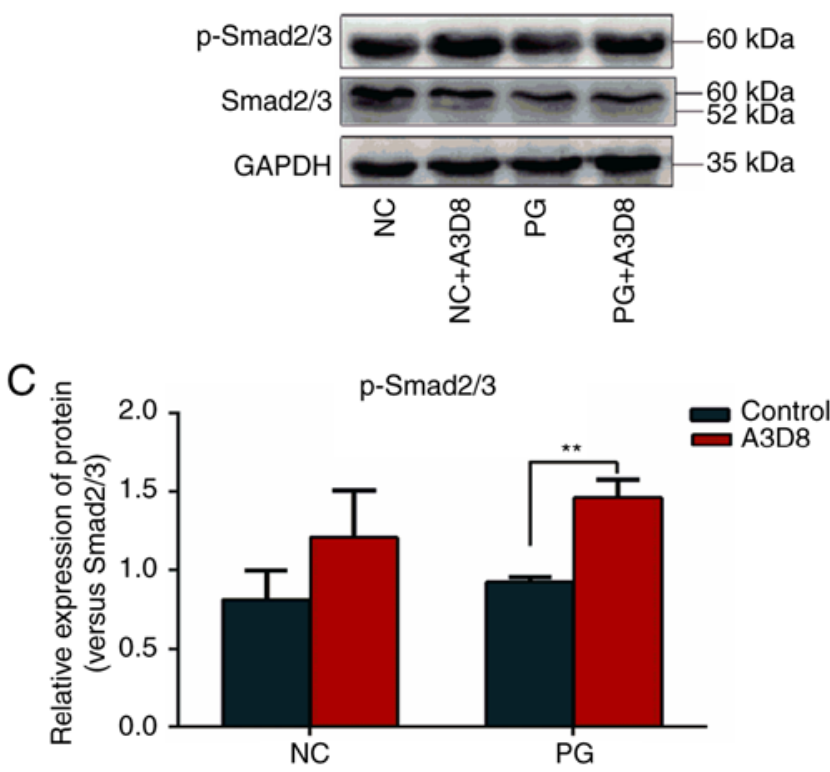

E

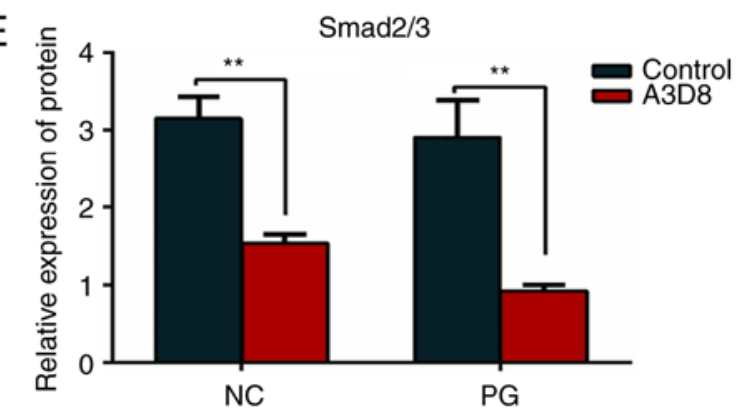

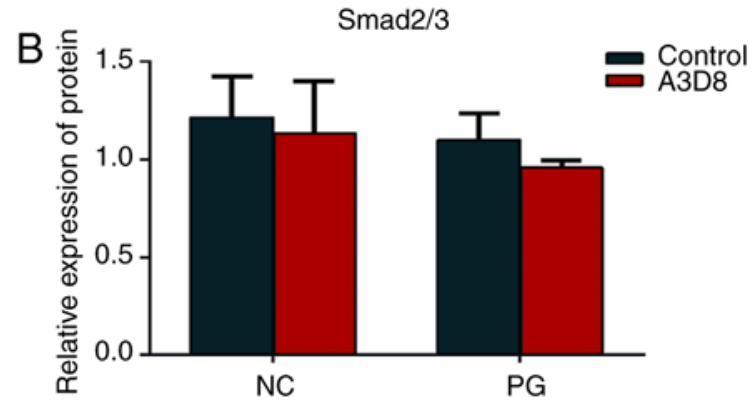
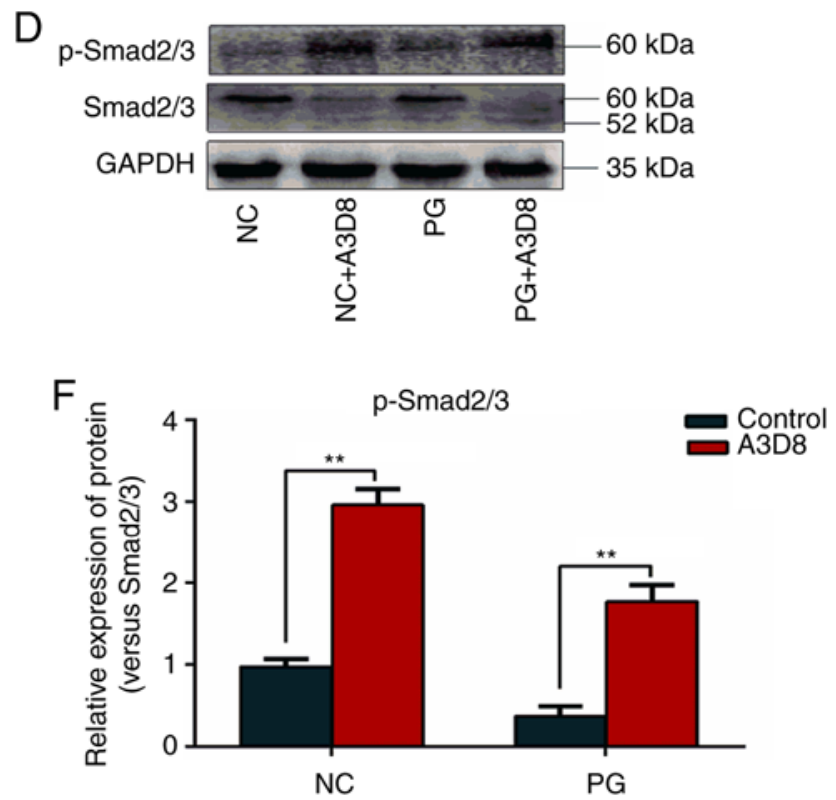

Figure 5. Suppression of CD44 enhances Smad2/3 signaling during the chondrogenic differentiation of hAMSCs. (A) Expression levels of p-Smad2/3 and Smad2/3 proteins. (B) Relative expression of total Smad2/3 and (C) ratio of p-Smad2/3 to Smad2/3 on day 1 after treatment with A3D8. (D) Expression levels of p-Smad2/3 and Smad2/3 proteins. Relative expression levels of (E) total Smad2/3 and (F) ratio of p-Smad2/3 to Smad2/3 on day 3 after treatment with A3D8. Data were presented as mean \pm standard errors of the mean $(n=3) .{ }^{* *} \mathrm{P}<0.01$. CD, cluster of differentiation; hAMSCs, human amniotic mesenchymal stem cells; p-, phosphorylated; NC, negative control group; PG, positive drug group.

that CD44 served an important role during the differentiation of hAMSCs into chondrocytes. Suppression of CD44 could decrease the expression of cartilage-associated genes and inhibit the production of type II collagen and aggrecan, which are two important markers of chondrocytes (32). Furthermore, this process was closely associated with the ERK1/2 signaling pathway. These results suggested that CD44/ERK may be a key molecular hub for promoting the differentiation of hAMSCs into chondrocytes.

MSCs have been widely used as an alternative seed cell source for cartilage repair due to their chondrogenic differentiation potential in benign conditions. The chondrogenic differentiation of MSCs is primarily dependent on chondrocyte marker genes, such as Sox9, Col2al and Acan. In the present study, hAMSCs (NC group) exhibited high expression of Col2 $\alpha 1$ and Acan and low expression for Sox9. Other MSCs derived from human adult bone marrow (33) or adipose (34) tissue did not express these chondrocyte marker genes prior to the induction of chondrogenic differentiation. In the present study, although hAMSCs in the NC group did not exhibit formation of type II collagen and aggrecan in the immunocytochemistry assay, other data were sufficient to suggest that hAMSCs exhibited stronger chondrogenic potential compared with other MSCs. In addition, Sox 9 was significantly elevated at transcriptional level in hAMSCs following the induction of chondrogenic differentiation. However, suppression of CD44 resulted in a significant decrease in expression level of Sox 9 in PG. As previously described, Sox 9 expression is associated with chondrocyte differentiation (35) and transfection of Sox 9 enhances the expression of Col2 $\alpha 1$ and Acan (36); Sox9 is an essential transcription factor for maintaining the cartilage phenotype and chondrogenesis (37).

CD44 is a transmembrane protein of cell adhesion molecules and is a well-known surface marker of human MSCs. The expression of CD44 at the transcriptional level was decreased significantly during the chondrogenic differentiation in the present study, and this was consistent with a previous study (32). However, CD44 is not a specific negative marker in chondrocytes and has been demonstrated to be highly expressed in human articular chondrocytes (15). Therefore, it is questionable whether CD44 could be used as a specific marker for 
human MSCs or chondrocytes. As aforementioned, CD44 is a receptor for hyaluronic acid and also serves an important role in cell differentiation. The signaling cascade triggered by the interaction between hyaluronic acid and CD44 significantly contributes to hyaluronic acid-induced chondrogenesis in human adipose-derived MSCs, while the suppression of hyaluronic acid interaction with CD44 decreases the chondrogenic differentiation of human adipose-derived MSCs (33). Another study demonstrated that dimerization of CD44 is responsive to hyaluronic acid and contributes to chondrogenic differentiation of human adipose-derived MSCs (16). Furthermore, the interaction between hyaluronic acid with CD44 in chondrocytes is also crucial to maintain cartilage (17). In the present study, suppression of CD44 significantly attenuated the production of extracellular matrix of chondrocytes including type II collagen and aggrecan during the differentiation of hAMSCs into chondrocytes and also led to a significant decrease in expression of chondrocyte-associated genes such as Sox $9, \operatorname{Col} 2 \alpha 1$ and Acan . However, these genes were also inhibited in the NC group following treatment with A3D8. Thus, it was hypothesized that CD44 may be expressed in both hAMSCs and chondrocytes and be altered due to the change in cellular microenvironment, such as the addition of an inducer; the underlying mechanism requires further investigation.

The differentiation of stem cells into chondrocytes involves multiple signaling pathways, such as TGF- $\beta / \mathrm{BMP}$, Wnt $/ \beta$-catenin and MAPKs (38-40). Certain TGF- $\beta$ superfamily members have been widely used to regulate cell differentiation by triggering a series of signaling cascades (41). A previous study showed that the 3-dimensional alginate culture of human MSCs in defined induction medium, without TGF- $\beta 3$, is not sufficient to fully develop hyaline cartilage-like constructs with lacunae formation (32), indicating that TGF- $\beta 3$ is a key component for the chondro-induction differentiation. Thus, TGF- $\beta 3$ was used to induce chondrogenic differentiation in hAMSCs in the present study.

CD44 is a downstream target gene of the $\mathrm{Wnt} / \beta$-catenin signaling pathway, which has been previously reported to be an important signaling pathway, implicated in the chondro-induction differentiation $(42,43)$. MAPK family transduction involves a multistep kinase cascade; ERK is a major kinase among MAPKs and has been shown to serve a critical role in mediating chondrogenesis and associated gene expression (29). Therefore, only the ERKs of MAPKs signaling pathway were examined in the present study. For example, during the differentiation of bone marrow mesenchymal stem cells into chondrocytes, the activation of ERK1/2 negatively regulates the differentiation of chondrocytes (44). In the present study, the transcription levels of ERK1 and ERK2 genes were downregulated in hAMSCs following CD44 inhibition and the level of ERK1/2 phosphorylation were also significantly decreased. However, the total ERK1/2 protein did not change with or without CD44 inhibitor treatment. Therefore, inhibition of CD44 attenuated the activity of ERK and ERK served a positive role during the chondro-induction differentiation of hAMSCs. As described previously, the exact role of ERK in chondrogenesis remains to be elucidated. For example, Bobick and Kulyk (44) reported that ERK signaling serves a negative role in cartilage-specific gene expression in embryonic limb mesenchyme, while a recent study indicated that ERK acts as a positive regulator of hyaluronic acid-induced chondrogenesis in adipose-derived stem cells (16).

Smad2/3 and ERK1/2 are the downstream molecules of TGF- $\beta$ and the major signaling mediator for modulating chondrogenesis (45). In addition, they show a cross-talk between Smad2/3 and ERK1/2 signaling during the regulation of the chondrogenic differentiation of MSC (28). The results of the present study indicated that $\mathrm{p}-\mathrm{Smad} 2 / 3$ expression was increased on days 1 and 3 following the suppression of CD44 and the ratio of $\mathrm{p}-\mathrm{Smad} 2 / 3$ to total $\mathrm{Smad} 2 / 3$ was also significantly upregulated. Notably, it was identified that the expression of total Smad2/3 was not equal in each group on day 3 and was different from the change in $\mathrm{p}-\mathrm{Samd} 2 / 3$. It was considered that CD44 inhibition may suppress the expression of $\mathrm{Smad} 2 / 3$, but it also promoted the phosphorylation of Smad2/3 through other pathways. A previous study reported that suppression of CD44 inhibited the expression of DUSP10/MKP5, a negative regulator of p38 MAPK and JNK pathways (46). Thus, CD44 inhibition could directly activate p38 MAPK and JNK to enhance the phosphorylation of protein, without being dependent on the regulation of total protein expression. The results of the present study confirmed our hypothesis. However, the association between ERK1/2 signaling and $\mathrm{Smad} 2 / 3$ signaling remains unclear. A previous study has reported that during the proliferation of smooth muscle cells, phosphorylation of ERK can be decreased following the use of Smad3 inhibitors (47). Conversely, Hough et al (48) identified that phosphorylation of ERK can regulate Smad signaling. The mechanism through which ERK and Smad interact remains to be elucidated.

In the present study, the expression of $\mathrm{p}-\mathrm{Smad} 2 / 3$ on day 3 was significantly decreased in the $\mathrm{NC}$ group compared with day 1 in PG. Similar results, that the expression of $\mathrm{p}-\mathrm{Smad} 2 / 3$ was gradually decreased in the early stage of chondrogenic differentiation, have also been reported (49). In the current study, a limitation was that only one time point was observed for 7 days and the whole process of cartilage differentiation was not monitored dynamically, so the role of CD44 during different stages was not fully understood. In addition, the role of CD44 for cartilage formation remains unknown in vivo. This is an interesting and complex question and will be further investigated in the near future.

In summary, the present study suggested that CD44 was a functional regulator of the differentiation of hAMSCs into chondrocytes by modulating the Smad2/3 and ERK1/2 signaling pathways. The results provided a potential novel strategy to enhance the capacity of MSCs to differentiate into chondrocytes.

\section{Acknowledgements}

Not applicable.

\section{Funding}

The authors received financial support from National Natural Science Foundation of China (grant no. 81660363), Science and Technology Innovation Leading Academics of National High-level Personnel of Special Support Program 
(grant no. GKFZ-2018-29), Guizhou High-Level Innovative Talent Support Program (grant no. QKH-RC-20154028) and Science and Technology Foundation of Guizhou (grant no. QKH-2017-1422).

\section{Availability of data and materials}

The datasets used and/or analyzed during the current study are available from the corresponding author on reasonable request.

\section{Authors' contributions}

YX wrote the manuscript. YX, YQW and ATW performed the experiments. YL and RML analyzed the data. YJZ performed the flow cytometry analysis. CYY and JHX designed the experiment, interpreted the data and modified the manuscript. All authors read and approved the final manuscript.

\section{Ethics approval and consent to participate}

The study and use of the human amniotic membrane were approved [approval no. (2014) 2-085] by the Ethics Committee of Affiliated Hospital of Zunyi Medical University (Zunyi, China). The sample was collected after obtaining written informed consent from the women.

\section{Patient consent for publication}

Not applicable.

\section{Competing interests}

The authors declare that they have no competing interests.

\section{References}

1. Hunziker EB: Articular cartilage repair: Basic science and clinical progress. A review of the current status and prospects. Osteoarthritis Cartilage 10: 432-463, 2002.

2. Caldwell KL and Wang J: Cell-based articular cartilage repair: The link between development and regeneration. Osteoarthritis Cartilage 23: 351-362, 2015.

3. Zuscik MJ, Hilton MJ, Zhang X, Chen D and O'Keefe RJ: Regulation of chondrogenesis and chondrocyte differentiation by stress. J Clin Invest 118: 429-438, 2008.

4. Barry FP and Murphy JM: Mesenchymal stem cells: Clinical applications and biological characterization. Int $\mathrm{J}$ Biochem Cell Biol 36: 568-584, 2004.

5. Uccelli A, Moretta L and Pistoia V: Mesenchymal stem cells in health and disease. Nat Rev Immunol 8: 726-736, 2008.

6. Richardson SM, Kalamegam G,Pushparaj PN, Matta C, Memic A, Khademhosseini A, Mobasheri R, Poletti FL, Hoyland JA and Mobasheri A: Mesenchymal stem cells in regenerative medicine: Focus on articular cartilage and intervertebral disc regeneration. Methods 99: 69-80, 2016.

7. Fellows CR, Matta C, Zakany R, Khan IM and Mobasheri A: Adipose, bone marrow and synovial joint-derived mesenchymal stem cells for cartilage repair. Front Genet 7: 213, 2016.

8. Kondo M, Yamaoka K and Tanaka Y: Acquiring chondrocyte phenotype from human mesenchymal stem cells under inflammatory conditions. Int J Mol Sci 15: 21270-21285, 2014.

9. Freitag J, Bates D, Boyd R, Shah K, Barnard A, Huguenin L and Tenen A: Mesenchymal stem cell therapy in the treatment of osteoarthritis: Reparative pathways, safety and efficacy-a review. BMC Musculoskelet Disord 17: 230, 2016.
10. Díaz-Prado S, Muiños-López E, Hermida-Gómez T, Rendal-Vázquez ME, Fuentes-Boquete I, de Toro FJ and Blanco FJ: Isolation and characterization of mesenchymal stem cells from human amniotic membrane. Tissue Eng Part C Methods 17: 49-59, 2011.

11. Nogami M, Tsuno H, Koike C, Okabe M, Yoshida T, Seki S, Matsui Y, Kimura T and Nikaido T: Isolation and characterization of human amniotic mesenchymal stem cells and their chondrogenic differentiation. Transplantation 93: 1221-1228, 2012.

12. Ranzoni AM, Corcelli M, Hau KL, Kerns JG, Vanleene M, Shefelbine S, Jones GN, Moschidou D, Dala-Ali B, Goodship AE, et al: Counteracting bone fragility with human amniotic mesenchymal stem cells. Sci Rep 6: 39656, 2016.

13. Muiños-López E, Hermida-Gómez T, Fuentes-Boquete I, de Toro-Santos J, Blanco FJ and Díaz-Prado SM: Human amniotic mesenchymal stromal cells as favourable source for cartilage repair. Tissue Eng Part A 23: 901-912, 2017.

14. Morath I, Hartmann TN and Orian-Rousseau V: CD44: More than a mere stem cell marker. Int J Biochem Cell Biol 81: $166-173,2016$.

15. Knudson CB: Hyaluronan and CD44: Strategic players for cell-matrix interactions during chondrogenesis and matrix assembly. Birth Defects Res C Embryo Today 69: 174-196, 2003.

16. Wu SC, Chen $\mathrm{CH}$, Wang JY, Lin YS, Chang JK and Ho ML: Hyaluronan size alters chondrogenesis of adipose-derived stem cells via the CD44/ERK/SOX-9 pathway. Acta Biomater 66: 224-237, 2018.

17. Grogan SP, Barbero A, Diaz-Romero J, Cleton-Jansen AM, Soeder S, Whiteside R, Hogendoorn PC, Farhadi J, Aigner T, Martin I and Mainil-Varlet P: Identification of markers to characterize and sort human articular chondrocytes with enhanced in vitro chondrogenic capacity. Arthritis Rheum 56: 586-595, 2007.

18. Sun Y, Liu WZ, Liu T, Feng X, Yang N and Zhou HF: Signaling pathway of MAPK/ERK in cell proliferation, differentiation, migration, senescence and apoptosis. J Recept Signal Transduct Res 35: 600-604, 2015.

19. Stanton LA, Underhill TM and Beier F: MAP kinases in chondrocyte differentiation. Dev Biol 263: 165-175, 2003

20. Wang Y, Luo S, Zhang D, Qu X and Tan Y: Sika pilose antler type I collagen promotes BMSC differentiation via the ERK1/2 and p38-MAPK signal pathways. Pharm Biol 55: 2196-2204, 2017.

21. Zhang F, Wang C, Lin J and Wang X: Oxidized low-density lipoprotein (ox-LDL) promotes cardiac differentiation of bone marrow mesenchymal stem cells via activating ERK1/2 signaling. Cardiovasc Ther 35: e12305, 2017.

22. Wang X, Xue Y, Ye W, Pang J, Liu Z, Cao Y, Zheng Y and Ding D: The MEK-ERK1/2 signaling pathway regulates hyaline cartilage formation and the redifferentiation of dedifferentiated chondrocytes in vitro. Am J Transl Res 10: 3068-3085, 2018.

23. Provot S, Nachtrab G, Paruch J, Chen AP, Silva A and Kronenberg HM: A-raf and B-raf are dispensable for normal endochondral bone development, and parathyroid hormone-related peptide suppresses extracellular signal-regulated kinase activation in hypertrophic chondrocytes. Mol Cell Biol 28: 344-357, 2008.

24. El-Hoss J, Kolind M, Jackson MT, Deo N, Mikulec K, McDonald MM, Little CB, Little DG and Schindeler A: Modulation of endochondral ossification by MEK inhibitors PD0325901 and AZD6244 (Selumetinib). Bone 59: 151-161, 2014.

25. Zhang LT, Liu RM, Luo Y, Zhao YJ, Chen DX, Yu CY and Xiao JH: Hyaluronic acid promotes osteogenic differentiation of human amniotic mesenchymal stem cells through the TGF- $\beta$ /Smad signaling pathway. Life Sci 232: 116669, 2019.

26. Magatti M, Pianta S, Silini A and Parolini O: Isolation, culture, and phenotypic characterization of mesenchymal stromal cells from the amniotic membrane of the human term placenta. Methods Mol Biol 1416: 233-244, 2016.

27. Livak KJ and Schmittgen TD: Schmittgen, Analysis of relative gene expression data using realtime quantitative PCR and the 2(-Delta Delta C(T)) method. Methods 25: 402-408, 2001.

28. Dominici M, Le Blanc K, Mueller I, Slaper-Cortenbach I, Marini F, Krause D, Deans R, Keating A, Prockop DJ and Horwitz E: Minimal criteria for defining multipotent mesenchymal stromal cells. The international society for cellular therapy position statement. Cytotherapy 8: 315-317, 2006. 
29. Li J, Zhao Z, Liu J, Huang N, Long D, Wang J, Li X and Liu Y: MEK/ERK and $\mathrm{p} 38$ MAPK regulate chondrogenesis of rat bone marrow mesenchymal stem cells through delicate interaction with TGF-beta1/Smads pathway. Cell Prolif 43: 333-343, 2010.

30. Liu RM, Sun RG, Zhang LT, Zhang QF, Chen DX, Zhong JJ and Xiao JH: Hyaluronic acid enhances proliferation of human amniotic mesenchymal stem cells through activation of Wnt/ $\beta$-catenin signaling pathway. Exp Cell Res 345: 218-229, 2016.

31. Knudson W and Loeser RF: CD44 and integrin matrix receptors participate in cartilage homeostasis. Cell Mol Life Sci 59: 36-44, 2002.

32. Schnabel M, Marlovits S, Eckhoff G, Fichtel I, Gotzen L, Vécsei V and Schlegel J: Dedifferentiation-associated changes in morphology and gene expression in primary human articular chondrocytes in cell culture. Osteoarthritis Cartilage 10: 62-70, 2002.

33. Lee HJ, Choi BH, Min BH and Park SR: Changes in surface markers of human mesenchymal stem cells during the chondrogenic differentiation and dedifferentiation processes in vitro. Arthritis Rheum 60: 2325-2332, 2009.

34. Wu SC, Chen CH, Chang JK, Fu YC, Wang CK Eswaramoorthy R, Lin YS, Wang YH, Lin SY, Wang GJ and Ho ML: Hyaluronan initiates chondrogenesis mainly via CD44 in human adipose-derived stem cells. J Appl Physiol (1985) 114: 1610-1618, 2013

35. Tew SR, Li Y, Pothacharoen P, Tweats LM, Hawkins RE and Hardingham TE: Retroviral transduction with SOX9 enhances re-expression of the chondrocyte phenotype in passaged osteoarthritic human articular chondrocytes. Osteoarthritis Cartilage 13: 80-89, 2005.

36. Kolettas E, Muir HI, Barrett JC and Hardingham TE: Chondrocyte phenotype and cell survival are regulated by culture conditions and by specific cytokines through the expression of Sox-9 transcription factor. Rheumatology (Oxford) 40: 1146-1156, 2001.

37. Bi W, Deng JM, Zhang Z, Behringer RR and de Crombrugghe B: Sox9 is required for cartilage formation. Nat Genet 22: 85-89, 1999.

38. Gómez-Leduc T, Desancé M, Hervieu M, Legendre F, Ollitrault D, de Vienne C, Herlicoviez M, Galéra P and Demoor M: Hypoxia is a critical parameter for chondrogenic differentiation of human umbilical cord blood mesenchymal stem cells in type I/III collagen sponges. Int J Mol Sci 18: pii: E1933, 2017.

39. Chowdhury TT, Salter DM, Bader DL and Lee DA: Signal transduction pathways involving p38 MAPK, JNK, NFkappaB and AP-1 influences the response of chondrocytes cultured in agarose constructs to IL-1beta and dynamic compression. Inflamm Res 57: 306-313, 2008.
40. Liu S, Zhang E, Yang M and Lu L: Overexpression of Wnt11 promotes chondrogenic differentiation of bone marrow-derived mesenchymal stem cells in synergism with TGF- $\beta$. Mol Cell Biochem 390: 123-131, 2014

41. Moustakas A, Pardali K, Gaal A and Heldin CH: Mechanisms of TGF-beta signaling in regulation of cell growth and differentiation. Immunol Lett 82: 85-91, 2002.

42. Deng Y, Lei G, Lin Z, Yang Y, Lin H and Tuan RS: Engineering hyaline cartilage from mesenchymal stem cells with low hypertrophy potential via modulation of culture conditions and Wnt/ß-catenin pathway. Biomaterials 192: 569-578, 2019.

43. Yuan X, Liu H, Huang H, Liu H, Li L, Yang J, Shi W, Liu W and $\mathrm{Wu} \mathrm{L}$ : The key role of canonical wnt//-catenin signaling in cartilage chondrocytes. Curr Drug Targets 17: 475-484, 2016.

44. Bobick BE and Kulyk WM: The MEK-ERK signaling pathway is a negative regulator of cartilage-specific gene expression in embryonic limb mesenchyme. J Biol Chem 279: 4588-4595, 2004.

45. Chen WH, Lo WC, Hsu WC, Wei HJ, Liu HY, Lee CH, Tina Chen SY, Shieh YH, Williams DF and Deng WP: Synergistic anabolic actions of hyaluronic acid and platelet-rich plasma on cartilage regeneration in osteoarthritis therapy. Biomaterials 35: 9599-9607, 2014.

46. Furuta J, Ariyoshi W, Okinaga T, Takeuchi J, Mitsugi S, Tominaga $\mathrm{K}$ and Nishihara T: High molecular weight hyaluronic acid regulates MMP13 expression in chondrocytes via DUSP10/MKP5. J Orthop Res 35: 331-339, 2017.

47. Shi J, Chen M, Ouyang L, Huang L, Lin X, Zhang W, Liang R, Lv Z, Liu S and Jiang S: Airway smooth muscle cells from ovalbumin-sensitized mice show increased proliferative response to TGF $\beta 1$ due to upregulation of Smad3 and TGF $\beta R I I$. J Asthma 54: 467-475, 2017.

48. Hough C, Radu M and Doré JJ: Tgf-beta induced Erk phosphorylation of smad linker region regulates smad signaling. PLoS One 7: e42513, 2012.

49. Dexheimer V, Gabler J, Bomans K, Sims T, Omlor G and Richter W: Differential expression of TGF- $\beta$ superfamily members and role of Smad1/5/9-signalling in chondral versus endochondral chondrocyte differentiation. Sci Rep 6: 36655, 2016.

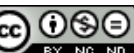

This work is licensed under a Creative Commons Attribution-NonCommercial-NoDerivatives 4.0 International (CC BY-NC-ND 4.0) License. 